\title{
Shift to earlier stage at diagnosis as a consequence of the National Bowel Cancer Screening Program
}

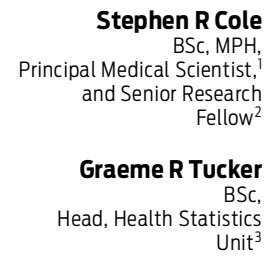

Joanne M Osborne $\mathrm{MPH}$,
Cancer Prevention Research Coordinator $^{2}$

Susan E Byrne BN,
Research Nurse

Peter A Bampton MD, FRACP, AGAF, Associate Professor

Robert J L Frase MB BS, FRACP, PhD, Professor of Gastroenterology2

Graeme P Young

MD, FRACP, FTSE

Professor of Global Gastrointestinal Health ${ }^{2}$

1 Bowel Health Service, Repatriation Genera Hospital, Adelaide, SA.

2 Flinders Centre for Innovation in Cancer

Flinders University.

Adelaide, SA.

3 Department of Health and Ageing, Adelaide, SA

steve.cole@

health.sa.gov.au

MJA 2013; 198: 327-330 doi: 10.5694/mjal2.11357

Editorial $\mathrm{p} 300$ tage at diagnosis is critical in determining the probability of survival with colorectal cancer (CRC). In randomised controlled trials, population screening using faecal occult blood tests (FOBT) results in earlier stage at diagnosis for screendetected cancers, ${ }^{1}$ and reduced mortality from colorectal malignancy compared with controls. ${ }^{2-4}$ Evaluations of cancer prevention programs with mortality as an end point take many years to complete. However, we know that early stage at diagnosis is linked to better prognosis and reduced mortality from CRC, so stage at diagnosis can serve as a surrogate marker for population mortality, and provides an early signal of program benefit.

After a pilot study in 2003, a faecal immunochemical test (FIT)-based National Bowel Cancer Screening Program (NBCSP) has been progressively rolled out across Australia. Participants in the program receive a free two-sample FIT kit by mail from a central register, collect samples and return them for testing. Results are mailed to participants and their nominated primary care practitioner (PCP). The PCP arranges follow-up of people with positive FIT results.

There is mandatory reporting of CRC in Australia, and the South Australian Cancer Registry (SACR) holds up-to-date records of CRC diagnoses in South Australia, including tumour stage. Thus the data held by the SACR and the NBCSP register records provide an opportunity to evaluate the effect of the NBCSP, as implemented in SA, on CRC stage at diagnosis.

Our primary aim was to determine whether CRCs diagnosed in people who had been invited to the NBCSP were diagnosed at an earlier stage than CRCs diagnosed in people not invited to the program. Our secondary aim was to determine whether downstaging was evident in the subpopulations that participated or that had positive test results in the screening program.

Abstract

Objective: To assess the impact of the National Bowel Cancer Screening Program (NBCSP) in South Australia.

Design, setting and participants: A cohort comparison of colorectal cancer (CRC) patient data from the NBCSP register and the South Australian Cancer Registry. Patient records of those invited to take part in screening through the NBCSP, those who participated in the program, and those with positive test results were compared with those of the rest of the study population (excluding the group of interest) on an intention-to-screen basis.

Main outcome measure: Stage of CRC at diagnosis as a surrogate marker for effect on CRC mortality.

Results: Of 3481 eligible patients, 221 had been invited to the NBCSP. Invitees were more likely to have stage A lesions compared with all other patients (34.8\% versus 19.2\%; $P<0.001$ ), and half as likely to have stage D CRC (5.4\% versus $12.4 \% ; P<0.001$ ). A further shift towards earlier stage was seen in those who participated in screening and those with positive test results compared with all other patients (38.8\% stage $A$ and $3.0 \%$ stage D in screening participants versus $19.3 \%$ stage $A$ and $12.4 \%$ stage $D$ in all other patients; and $39.7 \%$ stage $A$ and $2.6 \%$ stage $D$ in those with positive test results versus $19.3 \%$ stage $A$ and $12.4 \%$ stage $D$ in all other patients; $P<0.001$ ).

Conclusions: CRCs were diagnosed at a significantly earlier stage in people invited to the NBCSP compared with those who were not invited, regardless of participation status or test result. The NBCSP should lead to reductions in CRC mortality in Australia.

\section{Methods}

Patients were eligible for inclusion if they had CRC that had been reported to the SACR with a date of diagnosis between 1 January 2003 and 31 December 2008, and if they were aged 55-75 years at the date of diagnosis. This date and age range ensured inclusion of individuals invited to have a screening test in the NBCSP pilot program in SA (February 2003 to June 2004, with eligible participants aged 55-74 years on 1 January 2003) or in the NBCSP Phase I (22 January 2007 to 30 June 2008, with participants eligible if they turned 55 or 65 years of age in that period).

We compared the stage profiles of eligible patients invited to the NBCSP (invited), those who took up the offer to have a screening test (participant) and those who had positive results in the screening test (positive), relative to the stage profile of the study population excluding the group of interest (all other patients), on an intention-toscreen basis. Patients were allocated to the invited, participant and positive cohorts if their date of diagnosis was between 15 and 365 days from the date of invitation to participate in the NBCSP pilot program or Phase I trial. Finally, to gain some insight into the value of an invitation alone, we compared the stage profiles of patients who were invited to the NBCSP but did not participate in testing with those of patients who were not invited.

CRC stage was defined according to the Australian Clinico-Pathological Staging System (ACPS), with stages graded from $A$ to $D$ in order of increasing disease spread. ${ }^{5}$ Experienced SACR staff extracted ACPS stage from clinical reports. Where stage data were incomplete, additional information was sought from three public hospital-based cancer registries.

A list of invitees to both the NBCSP pilot program and Phase I trial was obtained from the NBCSP register. The Australian Institute of Health and Welfare carried out data-matching and provided a merged and de-identified dataset with, for each individual, 
1 Demographics of patients invited to the National Bowel Cancer Screening Program compared with those of all other patients in the study population

\begin{tabular}{lcc} 
Variable & $\begin{array}{c}\text { Invited patients } \\
n=221\end{array}$ & $\begin{array}{c}\text { All other patients } \\
n=3260\end{array}$ \\
\hline Sex* & $125(56.6 \%)$ & $1930(59.2 \%)$ \\
Male & $96(43.4 \%)$ & $1330(40.8 \%)$ \\
Female & $55(24.9 \%)$ & $525(16.1 \%)$ \\
Age ${ }^{\dagger}$ & $21(9.5 \%)$ & $671(20.6 \%)$ \\
$55-59$ years & $94(42.5 \%)$ & $864(26.5 \%)$ \\
$60-64$ years & $43(19.5 \%)$ & $988(30.3 \%)$ \\
$65-69$ years & $8(3.6 \%)$ & $212(6.5 \%)$ \\
$70-74$ years & $45(20.4 \%)$ & $720(22.1 \%)$ \\
$>74$ years & $27(12.2 \%)$ & $763(23.4 \%)$ \\
Area-level disadvantage by SEIFA quintile ${ }^{\ddagger}$ & $659(20.2 \%)$ \\
1 (most disadvantaged) & $49(22.2 \%)$ & $584(17.9 \%)$ \\
2 & $73(33.0 \%)$ & $534(16.4 \%)$ \\
3 & $27(12.2 \%)$ & \\
4 & $189(85.5 \%)$ & $2171(66.6 \%)$ \\
5 (least disadvantaged) & $473(14.5 \%)$ \\
Remoteness index ${ }^{5}$ (based on Accessibility/Remoteness Index \\
of Australia) \\
Urban & $13(5.9 \%)$ & $616(18.9 \%)$ \\
Rural & $19(8.6 \%)$ & \\
Remote &
\end{tabular}

SEIFA $=$ Socio-Economic Indexes for Areas.

$* \chi^{2}=0.60 ; P=0.434 . \dagger \chi^{2}=52.71 ; P<0.001 . \ddagger \chi^{2}=39.41 ; P<0.001$

$\S \chi^{2}=34.00 ; P<0.001$.

2 Distribution of colorectal cancer stage in patients (where stage was known; $n=3026$ ) according to whether they were invited to participate in the National Bowel Cancer Screening Program (NBCSP)*

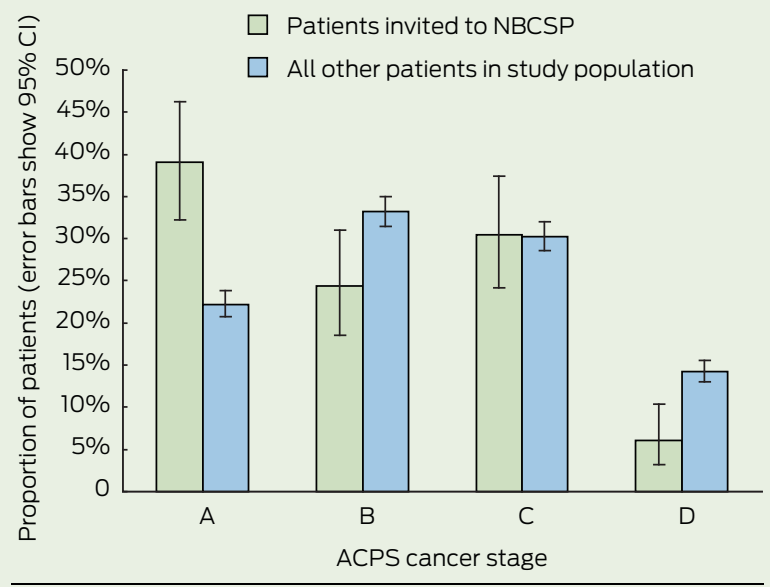

ACPS = Australian Clinico-Pathological Staging System

* Only patients with known colorectal cancer stage are included in sample shown; patients with missing or insufficient stage data are excluded.

CRC stage at diagnosis, NBCSP invitation status, NBCSP participation status, FIT result, age, sex, socioeconomic status (SES) and remoteness index data. Cohort stage profiles were compared by $\chi^{2}$ analyses. Multinomial logistic regression was performed using Stata version 12 (StataCorp) to control for possible differences between cohorts in age, sex, SES and geographical remoteness.

Ethics approval was obtained from the SA Health Human Research Ethics Committee and the Department of Health and Ageing Departmental Ethics Committee. Additional approvals were obtained from the Epidemiology Branch, SA Health, for access to the SACR; from the Royal Adelaide Hospital, Queen Elizabeth Hospital and Flinders Medical Centre for access to hospital-based registries; and from Medicare Australia for extracting data from the NBCSP register.

\section{Results}

We identified 3481 eligible patients with CRC reported to the SACR. Of these, 221 were allocated to the invited cohort. Staging data were available for $87.0 \%$ of patients: no data were available for $6.6 \%$, and a further $6.4 \%$ had insufficient data to determine ACPS stage. The invited cohort differed significantly from all other patients in age, SES and remoteness (Box 1 ).

\section{CRC stage according to invitation to the NBCSP}

The stage profiles of the invited cohort compared to the rest of the study population (where stage was known) are shown in Box 2. The difference in stage profiles was highly significant $\left(\chi^{2}=39.5 ; \quad P<0.001 ;\right.$ Box 3$)$. In the invited group, the percentage of stage A cancers was $34.8 \%$, versus $19.2 \%$ in all other patients $(P<0.001)$. Similarly, the percentage of stage $\mathrm{D}$ cancers was $5.4 \%$ in the invited group versus $12.4 \%$ in all other patients $(P=0.002)$.

There was a further shift towards earlier stage at diagnosis when the participant group was compared with all other patients $\left(\chi^{2}=47.7 ; P<0.001\right)$. In the participant group, the proportion with stage A was almost double that of all other patients $(38.8 \%$ versus $19.3 \%$; $P<0.001)$, while the percentage with stage $D$ was $3.0 \%$ versus $12.4 \%$ in all other patients $(P<0.001)$. This trend continued when the positive subgroup stage profile was compared with that of all other patients $\left(\chi^{2}=47.4 ; P<0.001\right)$. Of those in the participant group, 151/165 (91.5\%) returned a positive FIT result through the NBCSP. The percentage with stage A was twice that of all other patients $(39.7 \%$ compared with $19.3 \%$; $P<0.001)$, while the percentage with stage D was $2.6 \%$ compared with $12.4 \%$ in all other patients $(P<0.001)$.

Analyses that included or excluded patients with unknown cancer stage had no effect on the statistical significance of any of the findings. Multivariate analyses showed that age and SES were significantly associated with stage at diagnosis (Box 4). However, stage A lesions were significantly more likely to be diagnosed than stage $\mathrm{B}, \mathrm{C}$ or $\mathrm{D}$ CRC in the invited cohort relative to all other patients, while controlling for age, SES and remoteness. Stage A lesions were also more likely to be diagnosed in the participant and positive subgroups.

Finally, we compared the stage profiles of patients who were invited to the NBCSP but did not participate with the stage profiles of those who were not invited, to determine whether simply receiving an invitation but not participating led to downstaging. These groups did not differ in stage profile $\left(\chi^{2}=1.07 ; P=0.78\right)$.

\section{Discussion}

In this intention-to-screen analysisbased evaluation of the NBCSP, we found that CRCs diagnosed in people within 1 year of receiving an invitation to participate in the screening program were on average at an earlier stage than CRCs diagnosed in people who did not receive an invitation. There was a large and highly significant increase in stage A lesions and a corresponding decrease in stage $D$ CRC in those invited to the program relative to the rest of the study population, and the shift towards earlier stage progressively increased in participants in the screening test and in those who were recorded as having positive results in the FIT. Thus CRC downstaging was associated with an invitation to the NBCSP, and the strength of the effect increased in groups that excluded non-participants or people who had negative results in the FIT.

Downstaging was evident regardless of the inclusion or exclusion of patients with missing or insufficient data to determine staging. In addi- 
3 Colorectal cancer stage distribution of patients who were invited to, participated in or had positive test results in the National Bowel Cancer Screening Program (NBCSP), compared with the study population excluding the group of interest

\begin{tabular}{|c|c|c|c|c|c|c|}
\hline \multirow[b]{2}{*}{$\begin{array}{l}\text { ACPS cancer } \\
\text { stage }\end{array}$} & \multicolumn{2}{|c|}{$\begin{array}{l}\text { Patients invited to NBCSP } \\
\text { versus all other patients }\end{array}$} & \multicolumn{2}{|c|}{$\begin{array}{l}\text { Patients who participated in NBCSP } \\
\text { versus all other patients }\end{array}$} & \multicolumn{2}{|c|}{$\begin{array}{l}\text { Patients with positive test results in } \\
\text { NBCSP versus all other patients }{ }^{\ddagger}\end{array}$} \\
\hline & $\begin{array}{l}\text { Invited } \\
n=221\end{array}$ & $\begin{array}{l}\text { All other patients } \\
\qquad n=3260\end{array}$ & $\begin{array}{c}\text { Participant } \\
n=165\end{array}$ & $\begin{array}{l}\text { All other patients } \\
\qquad n=3316\end{array}$ & $\begin{array}{c}\text { Positive } \\
n=151\end{array}$ & $\begin{array}{l}\text { All other patients } \\
\qquad n=3330\end{array}$ \\
\hline No stage data & $9(4.1 \%)$ & $222(6.8 \%)$ & $5(3.0 \%)$ & $226(6.8 \%)$ & $5(3.3 \%)$ & $226(6.8 \%)$ \\
\hline $\begin{array}{l}\text { Insufficient data } \\
\text { to assess stage }\end{array}$ & 15 (6.8\%) & 209 (6.4\%) & 11 (6.7\%) & $213(6.4 \%)$ & $9(6.0 \%)$ & 215 (6.5\%) \\
\hline A & 77 (34.8\%) & $627(19.2 \%)$ & $64(38.8 \%)$ & $640(19.3 \%)$ & $60(39.7 \%)$ & 644 (19.3\%) \\
\hline B & $48(21.7 \%)$ & 941 (28.9\%) & 35 (21.2\%) & 954 (28.8\%) & $31(20.5 \%)$ & 958 (28.8\%) \\
\hline C & $60(27.1 \%)$ & 857 (26.3\%) & 45 (27.3\%) & $872(26.3 \%)$ & $42(27.8 \%)$ & 875 (26.3\%) \\
\hline D & $12(5.4 \%)$ & 404 (12.4\%) & $5(3.0 \%)$ & 411 (12.4\%) & $4(2.6 \%)$ & $412(12.4 \%)$ \\
\hline
\end{tabular}

ACPS = Australian Clinico-Pathological Staging System

$* \chi^{2}(5)=39.2 ; P<0.001 . \dagger \chi^{2}(5)=47.5 ; P<0.001 . \neq \chi^{2}(5)=47.4 ; P<0.001$

\begin{tabular}{|c|c|c|c|c|c|c|}
\hline \multirow[b]{2}{*}{ Variables } & \multicolumn{2}{|c|}{ ACPS stage B } & \multicolumn{2}{|c|}{ ACPS stage $C$} & \multicolumn{2}{|c|}{ ACPS stage $D$} \\
\hline & $\operatorname{RRR}(95 \% \mathrm{Cl})$ & $P$ & $\operatorname{RRR}(95 \% \mathrm{Cl})$ & $P$ & $\operatorname{RRR}(95 \% \mathrm{Cl})$ & $P$ \\
\hline Invited to NBCSP & $0.42(0.28-0.61)$ & 0.000 & $0.53(0.37-0.77)$ & 0.001 & $0.24(0.13-0.45)$ & 0.000 \\
\hline Age & $1.00(0.99-1.02)$ & 0.58 & $0.98(0.96-0.99)$ & 0.01 & $0.98(0.96-1.0)$ & 0.07 \\
\hline Male sex & $0.90(0.74-1.10)$ & 0.30 & $0.89(0.72-1.08)$ & 0.22 & $1.06(0.82-1.36)$ & 0.65 \\
\hline \multicolumn{7}{|c|}{ Area-level disadvantage by SEIFA quintile } \\
\hline 2 & $0.89(0.66-1.20)$ & 0.46 & $0.73(0.53-0.99)$ & 0.04 & $0.9(0.63-1.29)$ & 0.58 \\
\hline 3 & $0.79(0.58-1.07)$ & 0.13 & $0.86(0.64-1.17)$ & 0.35 & $0.57(0.39-0.84)$ & 0.01 \\
\hline 4 & $0.88(0.61-1.21)$ & 0.44 & $0.79(0.57-1.11)$ & 0.16 & $0.63(0.42-0.94)$ & 0.02 \\
\hline 5 (least disadvantaged) & $0.76(0.54-1.06)$ & 0.11 & $0.86(0.62-1.20)$ & 0.37 & $0.67(0.44-1.00)$ & 0.05 \\
\hline \multicolumn{7}{|c|}{ Remoteness index ${ }^{\dagger}$ (based on Accessibility/Remoteness Index of Australia) } \\
\hline Rural & $0.92(0.69-1.22)$ & 0.57 & $0.86(0.64-1.15)$ & 0.31 & $0.76(0.52-1.10)$ & 0.15 \\
\hline Remote and very remote & $1.06(0.80-1.39)$ & 0.69 & $1.12(0.85-1.47)$ & 0.44 & $0.96(0.68-1.34)$ & 0.80 \\
\hline
\end{tabular}

RRR = relative rate ratio. SEIFA = Socio-Economic Indexes for Areas.

* Relative to ACPS stage A base outcomes. † Relative to SEIFA 1.

tion, in a multivariate model, the relationship between early stage and screening through the NBCSP persisted when possible confounders age, SES and remoteness - were taken into account.

Earlier detection of CRC has a major impact on survival. United Kingdom data show 5-year relative survival rates of $>90 \%$ for Dukes' stage A cancer and $<7 \%$ for Dukes' stage D (Dukes' cancer stages are graded A-D in order of increasing spread and metastases). ${ }^{6}$ As randomised controlled trials have shown that CRCs detected through screening are diagnosed at an earlier stage, and screened populations had reduced mortality relative to control populations, ${ }^{1-4}$ it is valid to use downstaging as a surrogate for effect on mortality. The significantly earlier stage profile in patients who participated in the NBCSP should lead to reduced mortality rates in this population.
Although at the moment, only a relatively small proportion of the eligible Australian population is offered screening each year, the proposed gradual expansion of the NBCSP should result in greater reductions in CRC mortality over time, assuming that participation rates remain stable or increase.

Our findings are consistent with an earlier report using a hospital-based database of CRC patients, which showed an earlier stage distribution in people self-reporting that they were diagnosed through the NBCSP, compared with stage in symptomatic patients (ACPS stage I, 40\% in those diagnosed through the NBCSP versus $14 \%$ in non-participants; and stage IV, $3 \%$ in those diagnosed through the NBCSP versus $15 \%$ in non-participants). ${ }^{7}$ However, that study did not assess all CRCs diagnosed in the entire population. Further, the study was subject to recall bias and did not analyse results on an intention-toscreen basis. Our study included all cases of CRC reported to the SACR over the periods of implementation of the NBCSP pilot program and Phase I trial, and was based on an intentionto-screen analysis, which has allowed us to avoid sampling, temporal and follow-up quality bias.

Our results are also consistent with overseas evaluations of national CRC screening programs, although the methods used vary depending on the health system. The National Bowel Cancer Screening Programme in England reported a shift towards earlier stage disease in participants compared with patients with cancer diagnosed before the screening program. ${ }^{8}$ However, it is difficult to determine whether that downstaging represents improvement in practice over time or whether it was a direct result of the program. A decrease in the proportion of more advanced stage tumours for 
both men and women (but significant only in men) was also seen in the early stages of the English bowel cancer screening program, in a comparison of those who took up the offer of screening with those who did not, ${ }^{9}$ but the effect was not compared with stage distribution in patients diagnosed outside of the program. The Scottish CRC screening demonstration pilot study found a high proportion of cancers at Dukes' stage A (almost 50\%) when screening with guaiac faecal occult blood testing (gFOBT). ${ }^{10}$ A similar high proportion of stage A cancers was observed in the French pilot study. ${ }^{11}$ Unlike the overseas programs, Australia's NBCSP uses the FIT, and this is the first report of downstaging in a mass screening program using this testing method.

It was important to analyse the program in the first instance on an intention-to-screen basis as an impact at such a level demonstrates the value of the public health program and justifies its implementation.

This study has several strengths. Data were obtained from independently held and well managed databases, and individuals were matched across databases, and then de-identified by an independent third party, before analysis by the investigators. Selection bias was minimised, if not removed altogether, as it is unlikely that there was a difference between the proportions of CRCs reported to the SACR among NBCSP participants and the proportion reported among patients diagnosed outside of the program. All CRC diagnoses in the study population resulted from usual followup of patients after testing through the existing public and private primary care systems, and thus there were no systematic biases in the type of follow- up received by each cohort or in the time from referral to diagnosis. Additionally, stage data were extracted and interpreted by experienced SACR staff from histopathology reports. The cohorts examined had similar low proportions of patients with unknown CRC stage because of missing or insufficient data. Finally, this was a whole-of-population study that compared CRC stage at diagnosis of populations differing only in screening invitation status.

Although this is an observational study and it could be argued that other factors might have influenced stage, it was possible to adjust for a number of potential confounders. A second concern was that it was impossible to directly attribute an invitation to the NBCSP to a specific diagnosis of CRC. However, allocating patients to the invited cohort on the basis of a diagnosis between 14 and 366 days from the date of invitation is reasonable, considering the time taken for the clinical steps to final diagnosis after a positive test result; 14 days would appear to be the shortest time to a diagnosis. This timeline from the date of referral for colonoscopy to a diagnosis of CRC is consistent with results of studies across different health systems. ${ }^{12}$

\section{Conclusion}

In the context of a national CRC screening program with normal follow-up care for patients after testing, CRCs were diagnosed at a significantly earlier stage in people who had been invited to the program compared with people not invited to the program. Benefits were even greater in screening participants and those with positive results in the FIT. These results show that $\mathrm{CRC}$ screening works in practice and is likely to reduce CRC mortality in Australia.

Acknowledgements: This study was funded by the Department of Health and Ageing. The Department had no control or influence over the content of this report.

Competing interests: No relevant disclosures.

Received 5 Sep 2012, accepted 17 Jan 2013.

1 Hewitson P, Glasziou P, Irwig L, et al. Screening for colorectal cancer using the faecal occult blood test, Hemoccult. Cochrane Database Syst Rev 2007; (1): CD001216.

2 Mandel JS, Bond JH, Church TR, et al. Reducing mortality from colorectal cancer by screening for fecal occult blood. Minnesota Colon Cancer Control Study. N Engl J Med 1993; 328: 1365-1371.

3 Kronborg 0, Fenger C, Olsen J, et al. Randomised study of screening for colorectal cancer with faecal-occult-blood test. Lancet 1996;348: 1467-1471.

4 Hardcastle JD, Chamberlain JO, Robinson MH, et al. Randomised controlled trial of faecal-occultblood screening for colorectal cancer. Lancet 1996; 348: 1472-1477.

5 Davis NC, Newland RC. Terminology and classification of colorectal adenocarcinoma: the Australian clinico-pathological staging system. Aust N Z J Surg 1983; 53: 211-221.

6 National Cancer Intelligence Network. Colorectal cancer survival by stage - NCIN data debriefing. June 2009. http://www.ncin.org.uk/ publications/data_briefings/colorectal_cancer survival_by_stage.aspx (accessed Nov 2009).

7 Ananda SS, McLaughlin SJ, Chen F, et al. Initial impact of Australia's National Bowel Cancer Screening Program. Med J Aust 2009; 191: 378-381.

8 Ellul P, Fogden E, Simpson CL, et al. Downstaging of colorectal cancer by the National Bowel Cancer Screening Programme in England: first round data from the first centre. Colorectal Dis 2010; 12: 420-422.

9 Taylor EF, Morris EJA, Thomas JD, et al. Major improvement in the stage profile of tumours diagnosed in the NHS Bowel Cancer Screening Programme. Gut 2010; 59: A31. doi: 10.1136/ gut.2009.208975v.

10 Steele RJ, McClements PL, Libby G, et al. Results from the first three rounds of the Scottish demonstration pilot of FOBT screening for colorectal cancer. Gut 2009; 58: 530-535.

11 Goulard H, Boussac-Zarebska M, Ancelle-Park R, Bloch J. French colorectal cancer screening pilot programme: results of the first round. JMed Screen 2008; 15: 143-148.

12 Thompson MR, Heath I, SwarbrickET, et al. Earlier diagnosis and treatment of symptomatic bowel cancer: can it be achieved and how much will it improve survival? Colorectal Dis 2011;13:6-16. 口

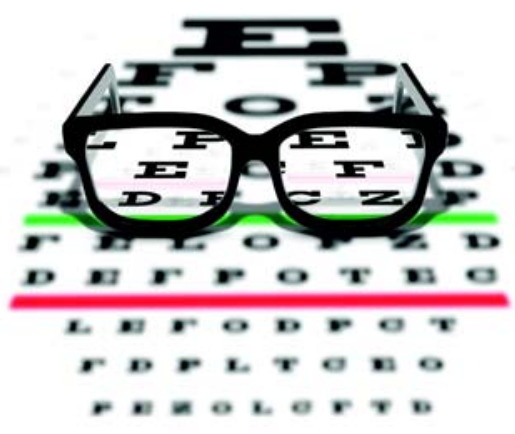

